\title{
Clinical effect of successful reperfusion in patients presenting with NIHSS < 8: data from the BEYOND-SWIFT registry
}

\author{
Johannes Kaesmacher ${ }^{1,2}$ (1) $\cdot$ Panagiotis Chaloulos-lakovidis ${ }^{2} \cdot$ Leonidas Panos $^{2} \cdot$ Pasquale Mordasini $^{1}$. \\ Mirjam R. Heldner ${ }^{2}$. Christoph C. Kurmann ${ }^{2}$ - Patrik Michel ${ }^{3}$. Steven D. Hajdu Marc Ribo $^{5}$ - Manuel Requena ${ }^{5}$. \\ Christian Maegerlein ${ }^{6} \cdot$ Benjamin Friedrich $^{6} \cdot$ Vincent Costalat $^{7} \cdot$ Amel Benali $^{7} \cdot$ Laurent Pierot $^{8} \cdot$ Matthias Gawlitza $^{8}$. \\ Joanna Schaafsma ${ }^{9} \cdot$ Vitor Mendes Pereira $^{10} \cdot$ Jan Gralla $^{1} \cdot$ Urs Fischer $^{2}$
}

Received: 4 September 2018 / Revised: 22 December 2018 / Accepted: 25 December 2018 / Published online: 8 January 2019

(c) The Author(s) 2019

\begin{abstract}
Background and purpose If patients presenting with large vessel occlusions (LVO) and mild symptoms should be treated with endvoascular treatment (EVT) remains unclear. Aims of this study were (1) assessing the safety and technical efficacy of EVT in patients with NIHSS $<8$ as opposed to a comparison group of patients presenting with NIHSS $\geq 8$ and (2) evaluation of the clinical effect of reperfusion in patients with NIHSS $<8$.

Methods Patients included into the retrospective multicenter BEYOND-SWIFT registry (NCT03496064) were analyzed. Clinical effect of achieving successful reperfusion (defined as modified Thrombolysis in Cerebral Infarction grade 2b/3) in patients presenting with NIHSS $<8(N=193)$ was evaluated using multivariable logistic regression analyses (displayed as adjusted Odds Ratios, aOR and $95 \%$ confidence intervals, 95\%-CI). Primary outcome was excellent functional outcome (modified Rankin Scale, mRS 0-1) at day 90. Safety and efficacy of mechanical thrombectomy in patients with NIHSS $<8$ was compared to patients presenting with NIHSS $\geq 8(N=1423)$.

Results Among patients with NIHSS $<8$ ( $N=193,77 / 193,39.9 \%$ receiving pre-interventional IV-tPA), successful reperfusion was significantly related to $\mathrm{mRS} 0-1$ (aOR 3.217, 95\%-CI 1.174-8.816) and reduced the chances of non-hemorrhagic neurological worsening (aOR 0.194, 95\%-CI 0.050-0.756) after adjusting for prespecified confounders. In interaction analyses, the relative merits of achieving successful reperfusion were mostly comparable between patients presenting with NIHSS $<8$ and NIHSS $\geq 8$ as evidenced by non-significantly different aOR. Interventional safety and efficacy metrics were similar between patients with NIHSS $<8$ and NIHSS $\geq 8$.

Conclusions Achieving successful reperfusion is beneficial in patients with persisting LVO presenting with NIHSS $<8$ and reduces the risk of non-hemorrhagic neurological worsening.
\end{abstract}

Keywords Mild symptoms $\cdot$ Mechanical thrombectomy $\cdot$ Endovascular $\cdot$ Low NIHSS $\cdot$ Thrombolysis $\cdot$ Stroke

\section{Introduction}

Jan Gralla and Urs Fischer contributed equally.

Electronic supplementary material The online version of this article (https://doi.org/10.1007/s00415-018-09172-1) contains supplementary material, which is available to authorized users.

Urs Fischer

urs.fischer@insel.ch

Extended author information available on the last page of the article
In a considerable proportion of stroke patients presenting with mild neurological symptoms, a proximal anterior circulation large-vessel occlusion (LVO) is identified as the underlying cause [1, 2]. However, in most patients with proximal LVO and low NIHSS scores on admission, indications for endovascular treatment (EVT) are not covered by current evidence derived from the large pivotal thrombectomy trials [3, 4]. In these patients, a well-developed collateral network presumably ensures sufficient blood flow to the territory distal to the occlusion site and neuronal functioning is largely maintained [5]. Intravenous thrombolysis 
(IVT) is the standard of care in patients presenting with mild disabling symptoms [6]. However, in patients with proximal LVO, IVT is often insufficient to recanalyze large clots and some patients deteriorate clinically [7-9] and tend to have poor outcomes if no reperfusion therapy is attempted, accordingly [10-12]. Hence, one of the most relevant unanswered questions is currently whether immediate EVT in patients with mild symptoms and LVO should be routinely considered to prevent clinical deterioration and to reduce infarct growth [11, 13-19]. While a proportion of patients will most likely benefit from routine EVT by preventing infarct growth, EVT also harbors the risk of worsening cerebral perfusion by thrombus dislocation and subsequent collateral shutdown and other procedural complications [20] (e.g. dissection [21], perforation [22], infarct to new territory [23], etc.).

The main aims of this registry analysis were (1) to assess safety and technical efficacy of EVT in LVO patients with NIHSS $<8$ when opposed to a large comparison group of patients with severe symptoms and (2) to evaluate the clinical effect of timely blood flow restoration in patients with NIHSS $<8$ as evidenced by measures of angiographical reperfusion success.

\section{Methods}

\section{BEYOND-SWIFT registry}

The Bernese-European RegistrY for ischemic stroke patients treated Outside current guidelines with Neurothrombectomy Devices using the SOLITAIRE ${ }^{\mathrm{TM}}$ FR With the Intention For Thrombectomy (BEYOND-SWIFT) is a retrospective, international, multicenter observational registry (https:// clinicaltrials.gov/ct2/show/NCT03496064). Inclusion criteria were (1) Treatment with a Medtronic market-released neurothrombectomy device (applied as initial devices used for intervention) in acute ischemic stroke patients. Patients were treated at the discretion of the investigator, independent of participation in this registry; (2) Patients or patient's legally authorized representatives have given informed consent according to Good Clinical Practices (GCP) and/or IRB and/or local or institutional policies allow use of observational registry data for research purposes.

Patients were excluded if they participated in another clinical trial or had withdrawn their consent for retrospective analyses. The following centers participated and contributed data of consecutive patients admitted to their hospital:

- Inselspital Bern, University Hospital Bern, University of Bern, Bern, Switzerland.

- CHUV, Lausanne University Hospital, Lausanne, Switzerland.
- Klinikum rechts der Isar, Technical University Munich, Munich, Germany.

- Montpellier CHU, University Hospital Montpellier, Montpellier, France.

- CHU Reims, University Hospital Reims, Reims, France.

- University Hospital Vall d'Hebron, Barcelona, Spain.

- Toronto Western Hospital-University Health Network, University of Toronto, Toronto, Canada.

An overview of included patients and rates of available follow-up data for each center can be found in Supplementary Table I. Ethical approval for inclusion of patient data in this pooled registry was obtained at each local responsible ethics committee (see Supplementary Table I). Additionally, ethical approval was obtained in Bern for pooling and anonymized analyses of the registry data (KEK Bern, Bern, Switzerland, Local Ethics Committee Study Identifier: 2018-00766).

Most patients included in the BEYOND-SWIFT $(n=2046)$ registry were treated for large-vessel anterior circulation strokes $(n=1820)$. Of these, 1630 had documented 90-day follow-up including 1616 with records of admission NIHSS. One-hundred-ninety-three of these patients presented with NIHSS $<8$ (11.9\%, see Fig. 1 for study flowchart). The corresponding lost-to-follow-up rate in the subgroup of patients with NIHSS $<8$ was $6.3 \%$ (13/206).

\section{Variables and image analysis}

The site of occlusion was categorized by local investigators into intracranial internal carotid artery, carotid-T/L, first/second/third segment of the middle cerebral artery (M1/M2/M3), first/second segment of the anterior cerebral artery (A1/A2), vertebral artery, basilar artery, or first/second segment of the posterior cerebral artery (P1/P2). For 7 patients, no data on occlusion site was available. Postinterventional modified thrombolysis in cerebral infarction (mTICI, 50\% or more reperfusion defined as TICI2b) scale was operator adjudicated at each center or rated by an independent research fellow, depending on the standard of the respective centers (see Supplementary Table I) [24]. Anterior circulation extracranial-intracranial tandem occlusion were defined as the presence of an intracranial LVO and $>90 \%$ cervical stenosis or occlusion. Of 1616 patients with anterior circulation LVO strokes, records of admission NIHSS and day 90 follow-up, 974 (60.3\%) underwent CT for admission workup, while 609 (37.7\%) patients received magnetic resonance imaging (MRI) including diffusionweighted sequences. For 33 patients $(2.0 \%)$ no information on admission imaging modality was provided. ASPECTS was evaluated at each site (see Supplementary Table I) and scores were available for 1518/1616 (93.9\%) patients in the whole cohort and in $184 / 193(95.3 \%)$ patients with 


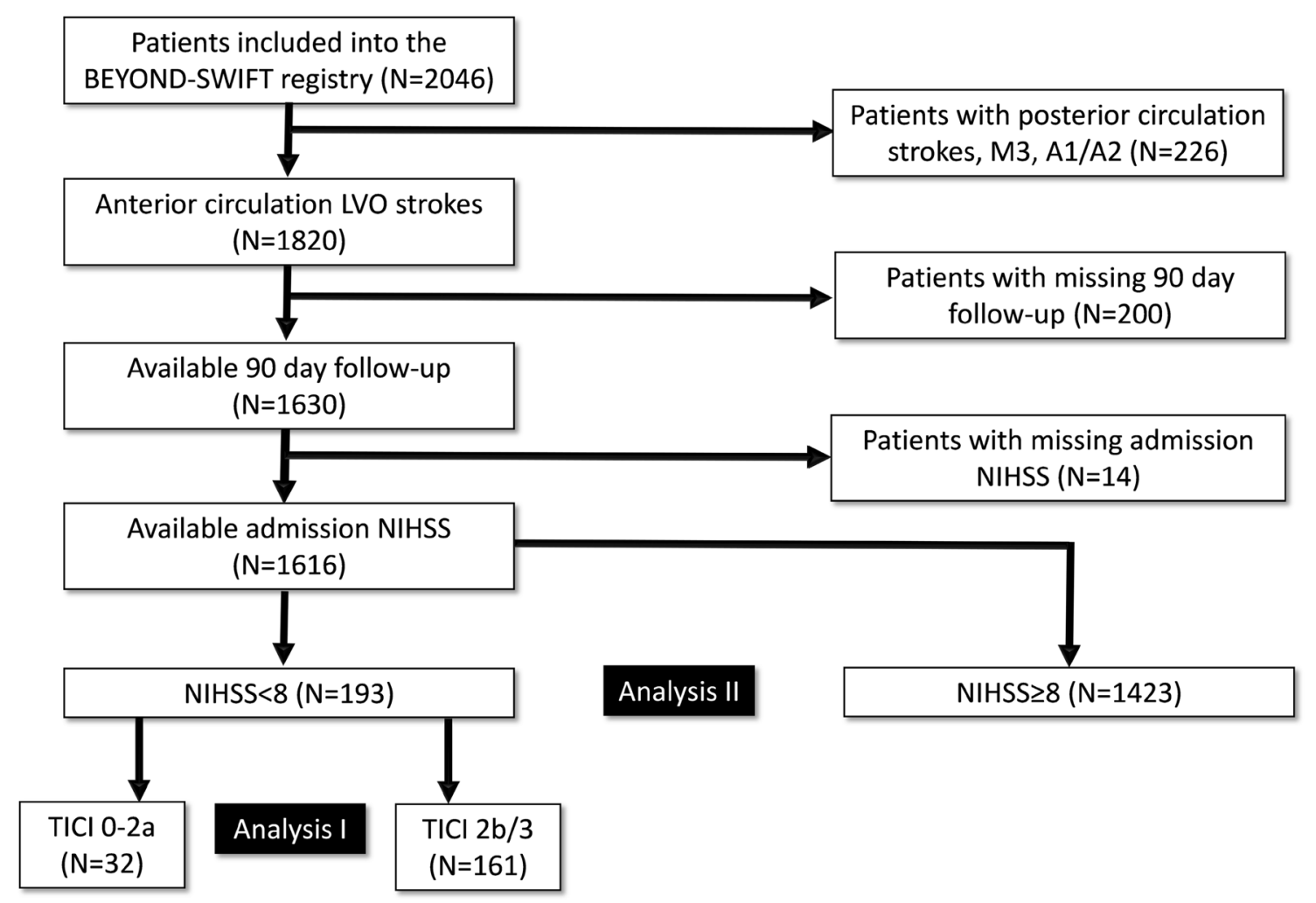

Fig. 1 Study Flow Chart of the BEYOND-SWIFT Registry

NIHSS $<8$ (including one patient with available ASPECTS but missing data on admission imaging modality). For clinical outcome evaluation, 3-month functional outcome was assessed applying the modified Rankin Scale (mRS) in routinely scheduled clinical visits or standardized telephone interviews, organized at each center. Rates of symptomatic intracerebral hemorrhage were reported by each center using the ECASS-II definition.

\section{Statistical analysis}

The primary endpoint of this analysis was mRS 0-1 (excellent outcome) at day 90 . Secondary and safety outcomes consisted of mRS 0-2 (good outcome) at day 90, all-cause mortality at day 90 , non-hemorrhagic neurological worsening and symptomatic intracerebral hemorrhage, which was assessed at each center applying the ECASS II criteria. Non-hemorrhagic neurological worsening was defined as drop in the NIHSS $\geq 4$ [25] between admission NIHSS and $24 \mathrm{~h}$ NIHSS without the occurrence of sICH [8]. Data on $24 \mathrm{~h}$ NIHSS were available for 1193/1616 in the complete cohort and 160/193 in the subcohort of patients with NIHSS $<8$. Note that in a comparison of no non-hemorrhagic neurological worsening vs non-hemorrhagic neurological worsening, patients with $\operatorname{sICH}(N=8)$ are excluded, leaving $N=152$ patients for final analysis in the subcohort of patients with NIHSS $<8$. Confidence intervals of proportions were calculated using the method outlined by Wilson, implementing a continuity correction [26]. Univariate comparisons between patients in whom reperfusion was successful (mTICI $2 b / 3$ ) and those in whom it was unsuccessful $(\mathrm{mTICI} \leq 2 \mathrm{a})$ were made using standard statistical measures (Fisher's exact test for categorical variables, Whitney-Mann $U$ Test for non-normally continuous or ordinally scaled variables and Welsch's $t$ test for independent normally distributed data). Association of successful reperfusion with all outcome parameters was assessed using multivariable logistic regression adjusting for the following pre-specified confounders: age (continuous), sex (categorical), NIHSS on admission (ordinal, adjusted odds ratio (aOR) per point increase), tandem vs non-tandem (categorical, tandem defined as $>90 \%$ cervical stenosis or cervical occlusion), center (categorical, contrast type: indicator, comparator: largest center), adjusted ASPECTS (see below, ordinal, adjusted odds ratio (aOR) per point increase), intravenous thrombolysis (categorical), risk factor hypertension (categorical), risk factor dyslipidemia (categorical), risk factor smoking (categorical), risk factor previous stroke (categorical), risk factor diabetes (categorical), in-hospital stroke (categorical), type of admission imaging (CT vs MRI, categorical), intracranial ICA/carotid-T vs M1 vs M2 occlusion 
(categorical, contrast type: indicator, comparator: ICA). To account for the imaging modality on admission (i.e. non-contrast CT vs DWI-MRI) on which the ASPECTS scoring was based upon, we increased all DWI-ASPECTS for regression analyses by 1 point according to the results derived from the SAMURAI registry [27]. For sensitivity purposes, analyses were rerun considering only patients with NIHSS $<6$ and also after additional implementation of the interaction term successful reperfusion * IVT.

\section{Results}

Of the 1616 patients included into this analysis, 193 patients had an initial NIHSS $<8$ (median NIHSS 5, IQR 4-6), comprising 103 patients with NIHSS $<6$. Distribution of the NIHSS scores in treated patients with NIHSS $<8$ is depicted in Supplementary Figure I. Patients with NIHSS $<8$ had better outcome than patients with NIHSS $\geq 8(N=1423)$, as evidenced by higher rates of excellent functional outcome (mRS $0-1,45.1 \%$ vs $25.6 \%, P<0.001$, see Fig. 2a) and lower mortality ( $16.1 \%$ vs $24.9 \%, P=0.007)$. However, rates of non-hemorrhagic neurological worsening tended to be higher in patients with NIHSS $<8$ as compared to patients with NIHSS $\geq 8(13.8 \%$ vs $9.0 \%, P=0.076)$.

In patients with NIHSS $<8$, there were no differences in baseline characteristics, comorbidities or treatment metrics when comparing patients with and without successful reperfusion (Table 1). Admission ASPECTS were not different between patients with successful and without successful reperfusion (MRI-DWI: median 9 vs $8, p=0.235$, CT: median 9 vs $9,0.248$, respectively). However, better outcomes were observed in patients with successful reperfusion as indicated by significantly higher rates of mRS $0-1$ (49.7\% vs $21.9 \%, P=0.006$, Fig. $2 b)$ and $\mathrm{mRS} 0-2(71.4 \%$ vs $37.5 \%, P<0.001)$. Median NIHSS improvement at $24 \mathrm{~h}$ was 3 (IQR 0-5) in successfully reperfusion patients and
-3 in non-successfully reperfused patients (IQR $-11-0$, $P<0.001$, Supplementary Figure II). The overall incidence of non-hemorrhagic neurological worsening in patients without $\mathrm{SICH}$ was $13.8 \%$ (95\%-CI 9.0\%-20.6\%), with significant differences among successfully reperfused patients and patients without successful reperfusion $(9.9 \%$ vs $38.1 \%$, $P=0.002)$. Rates of non-hemorrhagic neurological worsening were comparable among IVT and non-IVT pretreated patients $(12.5 \%$ vs $14.8 \%, P=0.813)$ and the reducing effect successful reperfusion on non-hemorrhagic neurological worsening was tangible also considering strata of IVT vs non-IVT patients (see Supplementary Table II). There was a trend towards lower mortality $(13.7 \%$ vs $28.1 \%, P=0.062)$ and lower rates of $\operatorname{sICH}(3.1 \%$ vs $9.4 \%, P=0.131)$ if successful reperfusion was achieved.

Among patients with NIHSS $<8$, successful reperfusion was a significant factor related to mRS $0-1$ (aOR 3.217, 95\%-CI 1.174-8.816, Table 2), mRS 0-2 (aOR 2.995, 95\%-CI 1.140-7.868) and reduced the chances of nonhemorrhagic neurological worsening (aOR 0.194, 95\%-CI $0.050-0.756)$ in multivariable binary logistic regression analysis adjusting for prespecified confounders outlined in the methods section. No significant associations were found for the endpoints sICH (aOR 0.086, 95\%-CI 0.006-1.234) and day 90 mortality (aOR $0.776,95 \%$-CI $0.240-2.509$ ), although point estimates favored reperfusion. No significant interactions between the above-mentioned associations and IVT pretreatment status were observed ( $P$ for interaction $>0.05$, see Table 2). The point estimates remained significant for mRS $0-1$ in a subgroup of patients with NIHSS $<6$, although uncertainty of the point estimates increased considerably (see Table 3 ). The relative merits of achieving successful reperfusion for various endpoints were comparable between patients presenting with NIHSS $<8$ and NIHSS $\geq 8$ (Fig. 3). However, reduction of mortality and sICH was found to be significant only in the cohort of patients presenting with NIHSS $\geq 8$. Here, a significant
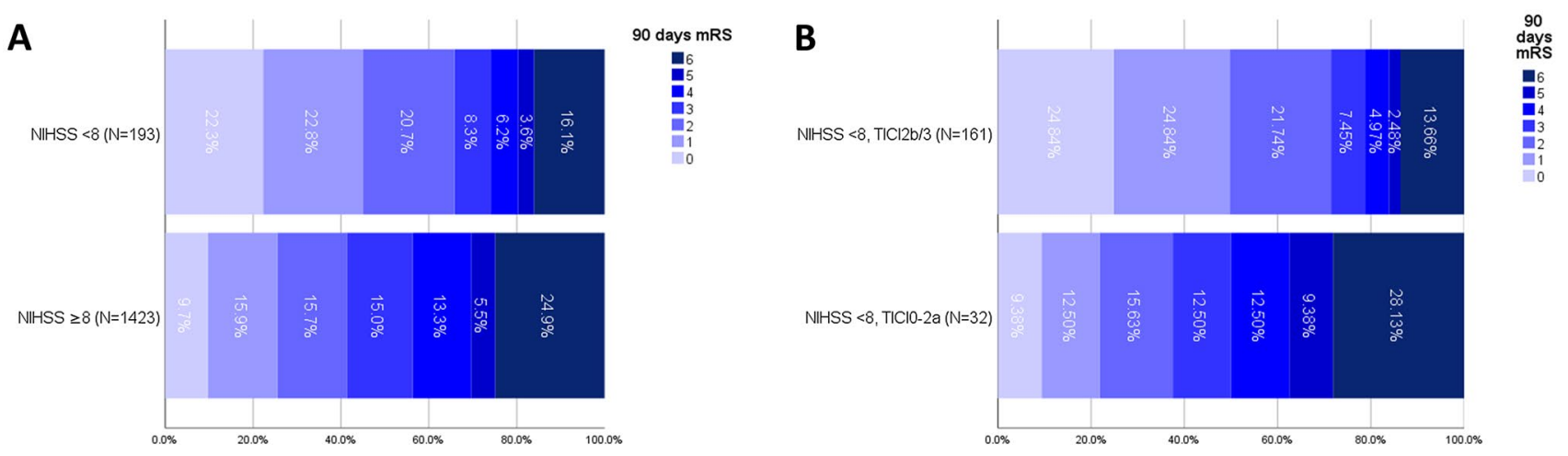

Fig. 2 Day 90 Functional Outcome. a Comparison of patients with NIHSS $<8$ and patients presenting with NIHSS $\geq 8$; b, Patients with NIHSS $<8$ dichotomized according to their reperfusion success; NIHSS National Institute of Health Stroke Scale, $m R S$ modified Rankin Scale 
Table 1 Patients with NIHSS $<8$ stratified according to reperfusion success (TICI0-2a vs TICI2b/3)

\begin{tabular}{|c|c|c|c|c|}
\hline NIHSS $<8, N=193$ & TICI0-3 $(N=193)$ & TICI0-2a $(N=32)$ & $\mathrm{TICI} 2 \mathrm{~b} / 3(N=161)$ & $P$ value \\
\hline Age (years) & $66.7+/-14.6$ & $68.2+/-13.1$ & $69.95+/-14.9$ & 0.503 \\
\hline Sex, female & $55.4 \%(107 / 193)$ & $53.1 \%(17 / 32)$ & $55.9 \%(90 / 161)$ & 0.846 \\
\hline Admission NIHSS & 5 (IQR 4-6) & 5 (IQR 3-6) & 5 (IQR 4-6) & 0.777 \\
\hline In-hospital stroke & $1.6 \%(3)$ & $0.0 \%(0 / 32)$ & $1.9 \%(3 / 161)$ & $>0.999$ \\
\hline Transfer & $36.5 \%(70 / 192)$ & $38.7 \%(12 / 31)$ & $36.0 \%(58 / 161)$ & 0.839 \\
\hline $\begin{array}{l}\text { Witnessed symptom-onset/last-seen well to admission } \\
(\min , N=178)\end{array}$ & $\begin{array}{r}148(\mathrm{IQR} \\
85-282)\end{array}$ & 201 (IQR 79-282, N=29) & $146(\mathrm{IQR} 87-285, N=149)$ & 0.769 \\
\hline $\begin{array}{l}\text { Witnessed symptom-onset/last-seen well to groin punc- } \\
\text { ture ( } \min , N=162)\end{array}$ & $\begin{array}{l}257 \text { (IQR } \\
196-393)\end{array}$ & $295(225-455, N=26)$ & $250(195-370, N=136)$ & 0.248 \\
\hline Admission Imaging, MRI $(N=191)$ & $57.6 \%(110 / 191)$ & $50.0 \%(16 / 32)$ & $59.1 \%(94 / 159)$ & 0.433 \\
\hline IVT & $39.9 \%(77 / 193)$ & $31.3 \%(10 / 32)$ & $41.6 \%(67 / 161)$ & 0.326 \\
\hline \multicolumn{5}{|l|}{ Risk factors } \\
\hline Smoking $(N=191)$ & $28.8 \%(55 / 191)$ & $18.8 \%(6 / 32)$ & $30.8 \%(49 / 159)$ & 0.203 \\
\hline Hypertension $(\mathrm{N}=193)$ & $60.1 \%(116 / 193)$ & $56.3 \%(18 / 32)$ & $60.9 \%(98 / 161)$ & 0.694 \\
\hline Dyslipidemia $(\mathrm{N}=192)$ & $56.3 \%(108 / 192)$ & $51.6 \%(16 / 31)$ & $57.1 \%(92 / 161)$ & 0.693 \\
\hline Previous CVE $(\mathrm{N}=191)$ & $12.6 \%(24 / 191)$ & $3.1 \%(1 / 32)$ & $14.5 \%(23 / 159)$ & 0.086 \\
\hline Diabetes $(N=193)$ & $13.5 \%(26 / 193)$ & $21.9 \%(7 / 32)$ & $11.8 \%(19 / 161)$ & 0.154 \\
\hline Occlusion site & & & & 0.203 \\
\hline iICA & $2.1 \%(4 / 193)$ & $3.1 \%(1 / 32)$ & $1.9 \%(3 / 161)$ & \\
\hline Carotid-T/L & $6.2 \%(12 / 193)$ & $12.5 \%(4 / 32)$ & $5.0 \%(8 / 161)$ & \\
\hline$M 1$ & $58.5 \%(113 / 193)$ & $46.9 \%(15 / 32)$ & $60.9 \%(98 / 161)$ & \\
\hline$M 2$ & $33.2 \%(64 / 193)$ & $37.5 \%(12 / 32)$ & $32.3 \%(52 / 161)$ & \\
\hline Extracranial-intracranial tandem occlusion & $15.5 \%(30 / 193)$ & $21.9 \%(7 / 32)$ & $14.3 \%(23 / 161)$ & 0.290 \\
\hline Underlying cervical dissection & $5.2 \%(10 / 193)$ & $6.3 \%(2 / 32)$ & $5.0 \%(8 / 161)$ & 0.673 \\
\hline TOAST $(N=192)$ & & & & 0.242 \\
\hline Large-artery & $9.4 \%(18 / 192)$ & $15.6 \%(5 / 32)$ & $8.1 \%(13 / 160)$ & \\
\hline Cardioembolism & $41.1 \%(79 / 192)$ & $28.1 \%(9 / 32)$ & $43.8 \%(70 / 160)$ & \\
\hline Other & $8.9 \%(17 / 192)$ & $12.5 \%(4 / 32)$ & $8.1 \%(13 / 160)$ & \\
\hline Unknown & $40.6 \%(78 / 192)$ & $43.8 \%(14 / 32)$ & $50.0 \%(64 / 160)$ & \\
\hline \multicolumn{5}{|l|}{ Outcome } \\
\hline mRS 0-1 & $45.1 \%(87 / 193)$ & $21.9 \%(7 / 32)$ & $49.7 \%(80 / 161)$ & $0.006 \dagger$ \\
\hline mRS 0-2 & $65.8 \%(127 / 193)$ & $37.5 \%(12 / 32)$ & $71.4 \%(115 / 161)$ & $<0.001 \dagger$ \\
\hline Non-hemorrhagic neurological worsening $(N=152)$ & $13.8 \%(21 / 152)$ & $38.1 \%(8 / 21)$ & $9.9 \%(13 / 131)$ & $0.002 \dagger$ \\
\hline Mortality & $16.1 \%(31 / 193)$ & $28.1 \%(9 / 32)$ & $13.7 \%(22 / 161)$ & 0.062 \\
\hline $\operatorname{sICH}(N=192)$ & $4.2 \%(8 / 192)$ & $9.4 \%(3 / 32)$ & $3.1 \%(5 / 160)$ & 0.131 \\
\hline
\end{tabular}

NIHSS National Institute of Health Stroke Scale, TICI Thrombolysis in Cerebral Infarction, IVT intravenous thrombolysis, CVE cerebrovascular event, $i I C A$ intracranial ICA, TOAST Trial of ORG 10,172 in Acute Stroke Treatment, $m R S$ modified Rankin Scale, IQR interquartile range $\dagger P<0.01$

interaction regarding the mortality reducing effect of successful reperfusion was found, suggesting the effect to be larger in patients presenting with NIHSS $\geq 8$ (Fig. 3).

When comparing patients with NIHSS $<8$ and patients with NIHSS $\geq 8$, a tendency for shorter groin-puncture to reperfusion intervals in patients with NIHSS $<8$ could be noted (median $42 \mathrm{~min}$ vs $47 \mathrm{~min}, P=0.075$ ). Apart from this, no differences in interventional safety and efficacy parameters were found (see Table 4). In particular rates of successful reperfusion $(83.4 \%$ vs $82.2 \%, P=0.690$ ), TICI3 reperfusion $(47.7 \%$ vs $44.4 \%, P=0.441)$ and complications $(11.4 \%$ vs $12.6 \%, P=0.818$ ) did not differ between patients presenting with mild symptoms and patients with NIHSS $\geq 8$.

\section{Discussion}

This registry-based retrospective analysis of consecutive patients treated with stent-retrievers has the following main findings: (1) Successful reperfusion is associated with better functional long-term outcome and early neurologic recovery in treated patients presenting with NIHSS $<8$. (2) The 
Table 2 Adjusted Odds ratios for TICI $2 \mathrm{~b} / 3$ for different outcome measures in patients with NIHSS $<8$

\begin{tabular}{llllll}
\hline NIHSS $<8 N=193$ & $\begin{array}{l}N \text { included in } \\
\text { the model }\end{array}$ & aOR TICI2b/3 & $95 \%$-CI & $P$ & $\begin{array}{c}P \text { for interac- } \\
\text { tion with IVT }\end{array}$ \\
\hline $\begin{array}{l}\text { Primary } \\
\text { mRS 0-1 }\end{array}$ & $179 / 193$ & 3.217 & $1.174-8.816$ & $0.023^{\dagger}$ & 0.343 \\
$\begin{array}{l}\text { Secondary } \\
\text { mRS 0-2 }\end{array}$ & $179 / 193$ & 2.995 & $1.140-7.868$ & $0.026^{\dagger}$ & 0.430 \\
sICH & $178 / 193$ & 0.086 & $0.006-1.234$ & 0.071 & 0.997 \\
$\begin{array}{l}\text { Mortality } \\
\text { Non-hemorrhagic neu- } \\
\text { rological worsening }\end{array}$ & $179 / 193$ & 0.776 & $0.240-2.509$ & 0.672 & 0.874 \\
\hline
\end{tabular}

NIHSS National Institute of Health Stroke Scale, aOR adjusted Odds Ratio, TICI Thrombolysis in Cerebral Infarction, 95\% CI 95\% confidence interval, IVT intravenous thrombolysis, $m R S$ modified Rankin Scale, sICH symptomatic intracerebral hemorrhage

${ }^{\dagger} P<0.05$

\begin{tabular}{|c|c|c|c|c|c|}
\hline NIHSS $<6 N=103$ & $\begin{array}{l}N \text { included in } \\
\text { the model }\end{array}$ & $\mathrm{aOR}$ TICI2b/3 & $95 \%-\mathrm{CI}$ & $P$ & $\begin{array}{l}P \text { for interac- } \\
\text { tion with } \\
\text { IVT }\end{array}$ \\
\hline \multicolumn{6}{|l|}{ Primary } \\
\hline mRS 0-1 & $98 / 103$ & 4.878 & $1.196-19.889$ & $0.027 \dagger$ & 0.934 \\
\hline \multicolumn{6}{|l|}{ Secondary } \\
\hline mRS 0-2 & $98 / 103$ & 3.690 & $0.911-14.949$ & 0.067 & 0.795 \\
\hline sICH & $97 / 103$ & Did not converge & - & - & - \\
\hline Mortality & $98 / 103$ & 1.068 & $0.180-6.343$ & 0.942 & 0.936 \\
\hline $\begin{array}{l}\text { Non-hemorrhagic neu- } \\
\text { rological worsening }\end{array}$ & $77 / 103$ & 0.111 & $0.011-1.083$ & 0.059 & 0.149 \\
\hline
\end{tabular}

NIHSS National Institute of Health Stroke Scale, aOR adjusted Odds Ratio, TICI Thrombolysis in Cerebral Infarction, 95\% CI 95\% confidence interval, IVT intravenous thrombolysis, $m R S$ modified Rankin Scale, sICH symptomatic intracerebral hemorrhage

${ }^{\dagger} P<0.05$ effect remained tangible in the subgroup of patients with NIHSS $<6$, however, there is large uncertainty according to the small sample size. (3) When compared with patients presenting with severe symptoms, interventional procedures in patients with NIHSS $<8$ were equally safe and technically effective and the relative merit of successful reperfusion on promoting good outcome was comparable.

Irrespective of treatment modalities, patients with low NIHSS and large-vessel occlusion have a more benign course than their counterparts presenting with severe neurological deficits [28]. Several underlying factors are causal for this observation, including incomplete occlusion [29, 30], partially permeable thrombi [31], and excellent collaterals, all of which allow for an adequately maintained blood flow to the brain tissue located distally to the LVO. However, there is compelling evidence that reperfusion therapies (IVT and/or EVT) improve the outcome of LVO patients also if they present only with mild neurological symptoms [11, 32-34]. Still, around 10-30\% of patients will deteriorate after IVT treatment, which is a phenomenon related to lysis-refractoriness, "collateralfailure" [35-39] and thrombus extension [40]. Correspondingly, nearly one-quarter of patients primarily treated with medical therapy do not achieve functional independence [33] and $>60 \%$ of patients without successful reperfusion remained functionally depend in the presented cohort. As long as those patients-likely to deteriorate-cannot precisely be identified a priori, there are two general treatment approaches, both having their advantages and disadvantages: (1) Subjecting all patients with acute LVO to EVT, thus putting patients at interventional risk, who would have reperfused spontaneously or after IVT treatment at comparable time points; (2) Only subject patients to EVT if they experience clinical deterioration, implying that reperfusion will be achieved later and risking that additional tissue will undergo infarction. A recent multicenter study comparing both of these treatment approaches on a center-level basis, found that patients undergoing either emergent mechanical EVT or delayed EVT in case of secondary clinical deterioration had comparable 


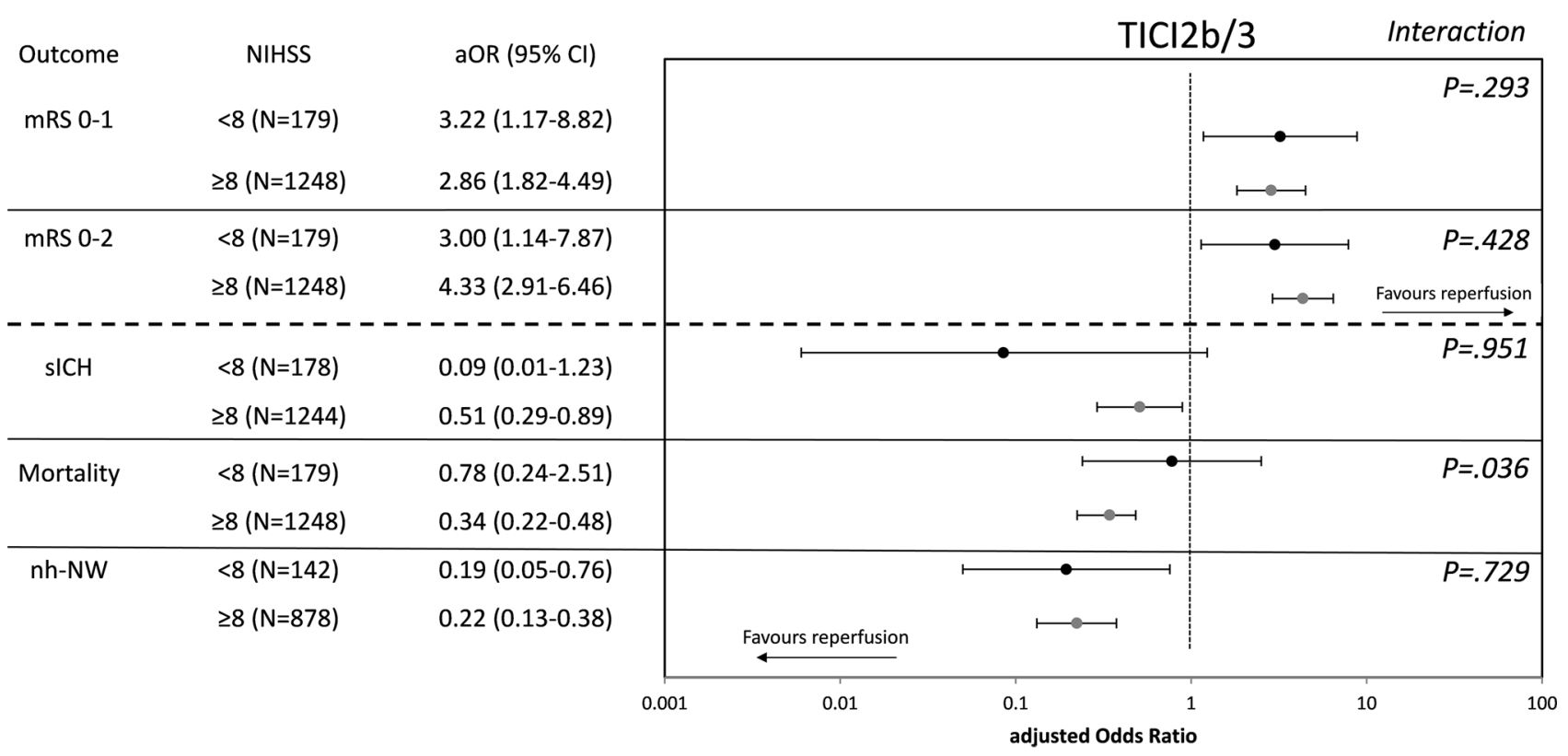

Fig. 3 adjusted Odds Ratios of successful reperfusion (TICI2b/3) for various endpoints with strata of admission NIHSS $<8$ vs NIHSS $\geq 8$. Adjusted Odds Ratios were calculated in split cohorts using multivariable binary logistic regression adjusting for all variables outlined in the methods section. Analysis was rerun implementing the variable
NIHSS $<8$ vs NIHSS $\geq 8$ and the term NIHSS $<8$ vs NIHSS $\geq 8 *$ $\mathrm{TICI} 2 \mathrm{~b} / 3$ (reperfusion) to test for potential interaction in the whole cohort (the variable admission NIHSS was ommitted in this model, accordingly)

Table 4 Comparison of technical efficacy and safety in patients with NIHSS $<8$ compared to patients with NIHSS $\geq 8$

\begin{tabular}{lll}
\hline $\begin{array}{l}\text { All anterior circulation LVO strokes with available mRS } \\
\text { and NIHSS }(N=1621)\end{array}$ & NIHSS $<8(N=193)$ & NIHSS $\geq 8(N=1423)$ \\
\hline Successful reperfusion & $83.4 \%(161 / 193)$ & $82.2 \%(1169 / 1422)$ \\
TICI3 & $47.7 \%(92 / 193)$ & $44.4 \%(633 / 1422)$ \\
Number of maneuvers $(N=1147)$ & $1(\mathrm{IQR} 1-2, N=158)$ & $2(\mathrm{IQR} 1-3, N=984)$ \\
Time from groin puncture to reperfusion $($ min, $N=1507)$ & $42(\mathrm{IQR} 29-65, N=184)$ & $47(\mathrm{IQR} 30-78, N=1323)$ \\
Other devices used as rescue $(N=1338)$ & $8.5 \%(14 / 164)$ & $12.1 \%(142 / 1169)$ \\
Complications & $11.4 \%(23 / 193)$ & $12.6 \%(179 / 1420)$ \\
Type of complications (relative frequency) & & \\
Vasospasms & $30.4 \%(7 / 23)$ & $24.7 \%(44 / 178)$ \\
Dissection & $34.8 \%(8 / 23)$ & $16.9 \%(30 / 178)$ \\
Perforation & $13.0 \%(3 / 23)$ & $11.8 \%(21 / 178)$ \\
Other & $13.0 \%(3 / 23)$ & $14.6 \%(26 / 178)$ \\
ENT & $8.7 \%(2 / 23)$ & $30.9 \%(55 / 178)$ \\
Multiple & $0 \%(0 / 23)$ & $1.1 \%(2 / 178)$ \\
Missing information on type of complication & - & $N=1$ \\
sICH & $4.2 \%(8 / 192)$ & $6.0 \%(85 / 1417)$ \\
\hline
\end{tabular}

NIHSS National Institute of Health Stroke Scale, $m R S$ modified Rankin Scale, TICI Thrombolysis in Cerebral Infarction, ENT emboli to new territory, $\mathrm{sICH}$ symptomatic intracerebral hemorrhage

outcomes [28]. However, depending on the type of analysis, a trend towards better outcome in patients undergoing emergent EVT was noted [28]. A similar observation was made in a multi-center cohort described by Haussen et al.
Here, the authors found that mechanical thrombectomy in patients presenting with NIHSS $\leq 5$ was associated with higher rates of functional independence and a favorable NIHSS shift when compared to medical management 
alone [34]. These findings were corroborated by a recent meta-analysis suggesting improved outcome in patients with NIHSS $\leq 8$ if treated with thrombectomy as opposed to best medical treatment [41]. However, in this analysis, increased rates of sICH after endovascular therapy were found, warranting further data [41]. Importantly, in the presented cohort, the rates of sICH were markedly lower (4.2\%) than the proportion of patients experiencing sICH described in the meta-analysis (13.6\%) [41].

In line with our findings, several other single-arm EVT studies also found high rates of procedural success and good safety profiles of EVT in patients presenting with LVO and minor symptoms [18, 19, 42-44]. Corroborating our observations regarding the beneficial effect of successful reperfusion, Dargazanli et al. also reported that patients in whom TICI $2 \mathrm{~b}$ or TICI 3 could be achieved do significantly better, although uncertainty of point estimates was relatively large [18]. In addition to these results, we found no evidence that the effect of achieving successful reperfusion is different in patients treated with IVT and those with contraindication for IVT. While the presented data is insufficient to answer the questions regarding preferred treatment regimens in the subgroup of patients presenting with low NIHSS, the data suggest that routine EVT in this subgroup of patients may be beneficial, because it is very likely that successful and timely reperfusion will be achieved more often.

The rate of complications in patients with NIHSS $<8$ was comparable to recent meta-analysis data [20] and matches the frequency of complications observed in patients presenting with NIHSS $\geq 8$ in this registry. Two-thirds of complications were comprised by iatrogenic cervical dissections and periprocedural vasospasms, both associated with relatively low risk of prompting unfavorable outcomes [21]. When adjusting for the lower rates of successful reperfusion in patients with procedural complications, we did not observe an independent effect of complications on outcome (data not shown). This suggests that not achieving successful reperfusion, rather than the complication itself puts patients at risk for poor outcomes. Moreover, some reassurance is provided that the rates of non-hemorrhagic neurological worsening are unlikely to be higher than if treated with IVT only. In a recent analysis of the SITS registry dealing with minor strokes treated with IVT, non-hemorrhagic neurological worsening was found in $30 \%$ of ICA-T or Tandem occlusions, $16.7 \%$ of other ICA occlusion, $9.3 \%$ of $\mathrm{M} 1$ and $5.8 \%$ of M2 occlusions [8]. Transferring those frequencies to our study population with available $24 \mathrm{~h}$ NIHSS, a rate of $11.9 \%$ could be expected (Supplementary Table III), which is nonsignificantly less frequent than what we have observed in our cohort (13.8\%). It has to be kept in mind though, that early responders to IVT (unlikely to experience non-hemorrhagic neurological worsening) are a priori excluded in our cohort. Although the numbers were generally small, it should be stressed that more than every third patient with persistent occlusion on angiography in whom no successful reperfusion could be achieved experienced non-hemorrhagic neurological worsening in our cohort $(38.1 \%, 8 / 21)$. Nevertheless, it seems obvious that subjecting all patients with NIHSS $<8$ to EVT as a clinical routine will unavoidably put a minority of patients at risk for worsening of the perfusion status as compared to when endovascular treatment would have been withheld and our observation that non-hemorrhagic neurological worsening occurs more often in patients with low NIHSS supports this. Hence, it will need a randomized controlled trial to clarify if the potential benefits of preventing natural course deterioration outweighs the risks associated with procedural complications in patients who would otherwise stay clinically stable. Such a trial may also incorporate imaging selection criteria to identify patients with mild symptoms most likely to benefit [45]. In summary, the data presented stress that the effect of achieving successful reperfusion in the subgroup of patients with low NIHSS seems substantial and the comparable frequency of nonhemorrhagic neurological worsening between our cohort and findings derived from minor strokes patients included in the SITS registry further points towards safety equipoise in this subgroup of patients.

\section{Limitations}

This is a single-arm multicenter retrospective registry and has associated limitations. No comparison to patients treated with medical management only was performed. Several factors including final mTICI score and initial ASPECTS were not core-lab adjudicated but were rated at the respective centers. Subgroup analyses were generally confined to small cohorts, which introduces a large uncertainty of the presented effects as indicated by relatively wide confidence intervals. Moreover, interaction analyses may be underpowered. The available sample represents minor strokes with LVO in whom the exact reasons for this treatment decisions are not known and may thus do not present the whole population of minor strokes with LVO. Importantly, patients who reperfused before endovascular treatment were excluded from the registry. Hence, interaction analysis or comparisons of e.g. bridging vs direct mechanical thrombectomy patients will only cover patients, who had persistent occlusions on the first angiography run, or did not clinically improve after IVT, respectively. The lack of a significant difference or interaction thus does not imply that pre-interventional IVT is not beneficial in this subcohort of patients, because at least $10 \%-20 \%$ of patients will reperfuse before endovascular thrombectomy [46, 47], which has been associated with better clinical outcomes. 


\section{Conclusion}

Achieving successful reperfusion in patients with persistent LVO on first angiography runs is beneficial in patients presenting with NIHSS $<8$ and this effect is independent of whether patients were pretreated with IVT or not. Given the differences of the likelihood to achieve timely and complete reperfusion between best medical management and EVT, the presented data may be interpreted as a hint towards potential benefits of emergent routine EVT in this subgroup of patients. Randomized controlled trials comparing best medical treatment vs EVT in LVO patients with minor symptoms are warranted.

Funding The study was supported by Medtronic (Dublin, Ireland). Medtronic did not take part in the conception, design or manuscript draft of this study. The work of Dr. Kaesmacher was supported by the SAMW/Bangerter Foundation and the Swiss Stroke Society.

\section{Compliance with ethical standards}

Conflict of Interest Dr. Fischer is a consultant for Medtronic and Stryker and Co-PI of the SWIFT DIRECT trial (Medtronic). Dr Gralla is a global principal investigator of STAR (Solitaire FR Thrombectomy for Acute Revascularisation), clinical event committee member of the PROMISE study (European Registry on the ACE Reperfusion Catheters and the Penumbra System in the Treatment of Acute Ischemic Stroke; Penumbra), and a principal investigator and consultant for the SWIFT DIRECT study (Medtronic) and receives Swiss National Science Foundation (SNSF) grants for magnetic resonance imaging in stroke. Dr Pierot serves as a consultant for Balt, Microvention, and Penumbra. Dr. Kaesmacher has received travel grants from Pfizer and Stryker and received research grants from the SAMW/Bangerter Foundation and the Swiss Stroke Society. Dr Ribo serves as a consultant for Medtronic, Stryker, Anaconda, Apta Targets, and Perflow Medical and as a speaker for Neuravi. Dr Michel has received funding for speaker honoraria from Boehringer. He has served on scientific advisory boards also for Boehringer. He has received research grants from Bristol-Myers Squibb, Boehringer, and the Swiss Heart Foundation. Dr. Pereira is a consultant for Stryker (SC for DAWN trial), Penumbra (SC for PROMISE study), BALT (proctorship of products unrelated to ischemic stroke), Phenox, Rapid Medical, Neurovasc and receives research a grant from Philips. All other authors declare that there are no conflicts of interest.

OpenAccess This article is distributed under the terms of the Creative Commons Attribution 4.0 International License (http://creativeco mmons.org/licenses/by/4.0/), which permits unrestricted use, distribution, and reproduction in any medium, provided you give appropriate credit to the original author(s) and the source, provide a link to the Creative Commons license, and indicate if changes were made.

\section{References}

1. Heldner MR, Zubler C, Mattle HP et al (2013) National institutes of health stroke scale score and vessel occlusion in 2152 patients with acute ischemic stroke. Stroke 44:1153-1157. https://doi. org/10.1161/STROKEAHA.111.000604
2. Maas MB, Furie KL, Lev MH et al (2009) National Institutes of Health Stroke Scale score is poorly predictive of proximal occlusion in acute cerebral ischemia. Stroke 40:2988-2993. https://doi. org/10.1161/STROKEAHA.109.555664

3. Goyal M, Menon BK, Van Zwam WH et al (2016) Endovascular thrombectomy after large-vessel ischaemic stroke: a meta-analysis of individual patient data from five randomised trials. Lancet 387:1723-1731. https://doi.org/10.1016/S0140-6736(16)00163-X

4. Powers WJ, Rabinstein AA, Ackerson T et al (2018) 2018 Guidelines for the early management of patients with acute ischemic stroke: a guideline for healthcare professionals from the american heart association/american stroke association. Stroke 49(3):e46e110. https://doi.org/10.1161/STR.0000000000000158

5. Marks MP, Lansberg MG, Mlynash M et al (2014) Effect of collateral blood flow on patients undergoing endovascular therapy for acute ischemic stroke. Stroke 45:1035-1039. https://doi. org/10.1161/STROKEAHA.113.004085

6. Emberson J, Lees KR, Lyden P et al (2014) Effect of treatment delay, age, and stroke severity on the effects of intravenous thrombolysis with alteplase for acute ischaemic stroke: a metaanalysis of individual patient data from randomised trials. Lancet 384:1929-1935. https://doi.org/10.1016/S0140-6736(14)60584-5

7. Kim JT, Park MS, Chang J et al (2013) Proximal arterial occlusion in acute ischemic stroke with low NIHSS scores should not be considered as mild stroke. PLoS One 8:1-7. https://doi. org/10.1371/journal.pone.0070996

8. Mazya MV, Cooray C, Lees KR et al (2017) Minor stroke due to large artery occlusion. When is intravenous thrombolysis not enough? Results from the SITS international stroke thrombolysis register. Eur Stroke J 3:239698731774600. https://doi. org/10.1177/2396987317746003

9. Rajajee V, Kidwell C, Starkman S et al (2006) Early MRI and outcomes of untreated patients with mild or improving ischemic stroke. Neurology 67:980-984. https://doi.org/10.1212/01. wnl.0000237520.88777.71

10. Mokin M, Masud MW, Dumont TM et al (2014) Outcomes in patients with acute ischemic stroke from proximal intracranial vessel occlusion and NIHSS score below 8. J Neurointerv Surg 6:413-417. https://doi.org/10.1136/neurintsurg-2013-010720

11. Griessenauer CJ, Medin C, Maingard J et al (2018) Endovascular mechanical thrombectomy in large-vessel occlusion ischemic stroke presenting with low national institutes of health stroke scale: systematic review and meta-analysis. World Neurosurg 110:263-269. https://doi.org/10.1016/j.wneu.2017.11.076

12. Nedeltchev K, Schwegler B, Haefeli T et al (2007) Outcome of stroke with mild or rapidly improving symptoms. Stroke, 38:2531

13. Messer MP, Schonenberger S, Mohlenbruch MA et al (2017) Minor stroke syndromes in large-vessel occlusions: mechanical thrombectomy or thrombolysis only? AJNR Am J Neuroradiol. https://doi.org/10.3174/ajnr.A5164

14. Urra X, Chamorro A (2013) Emerging issues in acute ischemic stroke. J Neurol 260:1687-1692. https://doi.org/10.1007/s0041 5-013-6919-x

15. Gallerini S, Marsili L, Bartalucci M et al (2017) Minor Stroke and thrombolysis: What is in the pipeline? AJNR Am J Neuroradiol 38:E84-E85. https://doi.org/10.3174/ajnr.A5298

16. Alqahtani SA, Stemer AB, McCullough MF et al (2017) Endovascular management of stroke patients with large vessel occlusion and minor stroke symptoms. Cureus 9:6-11. https://doi. org/10.7759/cureus. 1355

17. Xu G, Dong X, Niu X et al (2017) Cognitive function and prognosis of multimodal neuroimage-guided thrombectomy on mild to moderate anterior circulation infarction patients with broadened therapeutic window: a prospective study. Eur Neurol 257-263. https://doi.org/10.1159/000479735 
18. Dargazanli C, Consoli A, Gory B et al (2017) Is reperfusion useful in ischaemic stroke patients presenting with a low national institutes of health stroke scale and a proximal large vessel occlusion of the anterior circulation? Cerebrovasc Dis 43:305-312. https:// doi.org/10.1159/000468995

19. Haussen DC, Lima FO, Bouslama M et al (2017) Thrombectomy versus medical management for large vessel occlusion strokes with minimal symptoms: an analysis from STOPStroke and GESTOR cohorts. J Neurointerv Surg neurintsurg-2017-013243. 10:325-329, https://doi.org/10.1136/neurintsurg-2017-013243

20. Balami JS, White PM, Mcmeekin PJ et al (2017) Complications of endovascular treatment for acute ischemic stroke: prevention and management. 0:1-14. https://doi.org/10.1177/174749301774305 1

21. Goeggel Simonetti B, Hulliger J, Mathier E et al (2017) Iatrogenic vessel dissection in endovascular treatment of acute ischemic stroke. Clin Neuroradiol. https://doi.org/10.1007/s0006 2-017-0639-z

22. Mokin M, Fargen KM, Primiani CT et al (2016) Vessel perforation during stent retriever thrombectomy for acute ischemic stroke: technical details and clinical outcomes. J Neurointerv Surg Neurintsurg 2016-012707. https://doi.org/10.1136/neurintsurg-2016012707

23. Ganesh A, Al-Ajlan FS, Sabiq F et al (2016) Infarct in a new territory after treatment administration in the ESCAPE randomized controlled trial (endovascular treatment for small core and anterior circulation proximal occlusion with emphasis on minimizing ct to recanalization times). Stroke 47:2993-2998. https://doi. org/10.1161/STROKEAHA.116.014852

24. Higashida RT, Furlan AJ, Roberts H et al (2003) Trial design and reporting standards for intraarterial cerebral thrombolysis for acute ischemic stroke. J Vasc Interv Radiol 14:E1-E31. https:// doi.org/10.1016/S1051-0443(07)60431-X

25. Seners P, Turc G, Oppenheim C, Baron J (2015) Incidence, causes and predictors of neurological deterioration occurring within $24 \mathrm{~h}$ following acute ischaemic stroke: a systematic review with pathophysiological implications. J Neurol Neurosurg Psychiatry 86(1):87-94. https://doi.org/10.1136/jnnp-2014-308327

26. Newcombe RG (1998) Two-sided confidence intervals for the single proportion: comparison of seven methods. Stat Med 17:857872. doi: 10.1002/(SICI)1097-0258(19980430)17:8<857::AIDSIM777>3.0.CO;2-E

27. Nezu T, Koga M, Nakagawara J et al (2011) Early ischemic change on CT versus diffusion-weighted imaging for patients with stroke receiving intravenous recombinant tissue-type plasminogen activator therapy: stroke acute management with urgent risk-factor assessment and improvement (SAMURAI) rt-PA. Stroke 42:21962200. https://doi.org/10.1161/STROKEAHA.111.614404

28. Dargazanli C, Arquizan C, Gory B et al (2017) Mechanical thrombectomy for minor and mild stroke patients harboring large vessel occlusion in the anterior circulation a multicenter cohort study. Stroke 48:3274-3281. https://doi.org/10.1161/STROK EAHA.117.018113

29. Frölich AMJ, Psychogios MN, Klotz E et al (2012) Antegrade flow across incomplete vessel occlusions can be distinguished from retrograde collateral flow using 4-dimensional computed tomographic angiography. Stroke 43:2974-2979. https://doi. org/10.1161/STROKEAHA.112.668889

30. Maus V, You S, Kalkan A et al (2017) Incomplete large vessel occlusions in mechanical thrombectomy: an independent predictor of favorable outcome in ischemic stroke. Cerebrovasc Dis 44:113-121. https://doi.org/10.1159/000477499

31. Santos EMM, Marquering HA, den Blanken MD et al (2016) Thrombus permeability is associated with improved functional outcome and recanalization in patients with ischemic stroke. Stroke 47:732-741. https://doi.org/10.1161/STROKEAHA.115.011187
32. Heldner MR, Jung S, Zubler C et al (2014) Outcome of patients with occlusions of the internal carotid artery or the main stem of the middle cerebral artery with NIHSS score of less than 5: comparison between thrombolysed and non-thrombolysed patients. J Neurol Neurosurg Psychiatry 86(7):755-760. https:// doi.org/10.1136/jnnp-2014-308401

33. Haussen DiC, Bouslama M, Grossberg JA et al (2017) Too good to intervene? Thrombectomy for large vessel occlusion strokes with minimal symptoms: an intention-to-treat analysis. J Neurointerv Surg 9:917-921. https://doi.org/10.1136/neurintsurg-2016-01263 3

34. Haussen DC, Lima FO, Bouslama M et al (2018) Thrombectomy versus medical management for large vessel occlusion strokes with minimal symptoms: an analysis from STOPStroke and GESTOR cohorts. J Neurointerv Surg 10:325-329. https://doi. org/10.1136/neurintsurg-2017-013243

35. Bang OY, Saver JL, Buck BH et al (2008) Impact of collateral flow on tissue fate in acute ischaemic stroke. J Neurol Neurosurg Psychiatry 79:625-629. https://doi.org/10.1136/jnnp.2007.132100

36. Singer OC, Berkefeld J, Nolte CH et al (2015) Collateral vessels in proximal middle cerebral artery occlusion: the ENDOSTROKE study. Radiology 274:851-858. https://doi.org/10.1148/ radiol.14140951

37. Pham M, Bendszus M (2016) Facing time in ischemic stroke: an alternative hypothesis for collateral failure. Clin Neuroradiol 26:141-151. https://doi.org/10.1007/s00062-016-0507-2

38. Sallustio F, Motta C, Koch G et al (2017) Endovascular stroke treatment of acute tandem occlusion: a single-center experience. J Vasc Interv Radiol 28:543-549. https://doi.org/10.1016/j. jvir.2017.01.007

39. Campbell BCV, Christensen S, Tress BM et al (2013) Failure of collateral blood flow is associated with infarct growth in ischemic stroke. J Cereb Blood Flow Metab 33:1168-1172. https://doi. org/10.1038/jcbfm.2013.77

40. Seners $P$, Hurford R, Tisserand $M$ et al (2017) Is unexplained early neurological deterioration after intravenous thrombolysis associated with thrombus extension? Stroke 48:348-352. https://doi. org/10.1161/STROKEAHA.116.015414

41. Xiong Y, Gong J, Zhang Y et al (2018) Endovascular thrombectomy versus medical treatment for large vessel occlusion stroke with mild symptoms: a meta-analysis. PLoS One 13(8):e0203066. https://doi.org/10.1371/journal.pone.0203066

42. Pfaff J, Herweh C, Pham M et al (2016) Mechanical thrombectomy in patients with acute ischemic stroke and lower NIHSS scores: recanalization rates, periprocedural complications, and clinical outcome. Am J Neuroradiol 37:2066-2071. https://doi. org/10.3174/ajnr.A4862

43. Bhogal P, Bücke P, Ganslandt O et al (2016) Mechanical thrombectomy in patients with M1 occlusion and NIHSS score $\leq 5$ : a single-centre experience. Bmj 1:165-171. https://doi. org/10.1136/svn-2016-000052

44. Kaschner MG, Caspers J, Rubbert C et al (2018) Mechanical thrombectomy in MCA-mainstem occlusion in patients with low NIHSS scores. Interv Neuroradiol 24(4):398-404. https://doi. org/10.1177/1591019918760747

45. Bivard A, Lou M, Levi CR et al (2016) Too good to treat? Ischemic stroke patients with small computed tomography perfusion lesions may not benefit from thrombolysis. Ann Neurol 80:286-293. https://doi.org/10.1002/ana.24714

46. Seners P, Turc G, Naggara O et al (2018) Post-thrombolysis recanalization in stroke referrals for thrombectomy. Stroke 49:29752982. https://doi.org/10.1161/STROKEAHA.118.022335

47. Kaesmacher J, Giarrusso M, Zibold F et al (2018) Rates and quality of preinterventional reperfusion in patients with direct access to endovascular treatment. Stroke 49:1924-1932. https://doi. org/10.1161/STROKEAHA.118.021579 


\section{Affiliations}

Johannes Kaesmacher ${ }^{1,2}$ (1) $\cdot$ Panagiotis Chaloulos-lakovidis ${ }^{2} \cdot$ Leonidas Panos $^{2} \cdot$ Pasquale Mordasini $^{1}$. Mirjam R. Heldner ${ }^{2}$. Christoph C. Kurmann ${ }^{2}$ - Patrik Michel ${ }^{3}$. Steven D. Hajdu ${ }^{4}$ Marc Ribo $^{5}$ - Manuel Requena ${ }^{5}$. Christian Maegerlein ${ }^{6} \cdot$ Benjamin Friedrich $^{6} \cdot$ Vincent Costalat $^{7} \cdot$ Amel Benali $^{7}$. Laurent Pierot ${ }^{8} \cdot$ Matthias Gawlitza $^{8}$. Joanna Schaafsma ${ }^{9} \cdot$ Vitor Mendes Pereira $^{10} \cdot$ Jan Gralla ${ }^{1} \cdot$ Urs Fischer $^{2}$

1 University Institute of Diagnostic and Interventional Neuroradiology, University Hospital Bern, Inselspital, University of Bern, Bern, Switzerland

2 Department of Neurology, University Hospital Bern, Inselspital, University of Bern, Freiburgstrasse 8, 3010 Bern, Switzerland

3 Department of Neurology, CHUV Lausanne, Lausanne, Switzerland

4 Department of Radiology, CHUV Lausanne, Lausanne, Switzerland

5 Department of Neurology, Vall d'Hebron University Hospital, Barcelona, Spain
6 Department of Diagnostic and Interventional Neuroradiology, Klinikum rechts der Isar, Technical University Munich, Munich, Germany

7 Department of Neuroradiology, CHU Montpellier, Montpellier, France

8 Department of Neuroradiology, CHU Reims, Reims, France

9 Department of Neurology, Toronto Western Hospital, Toronto, ON, Canada

10 Joint Department of Medical Imaging, Toronto Western Hospital, Toronto, ON, Canada 